\section{In Reply: Abdominal- Transhiatal Resection for Adenocarcinoma of the Esophagogastric Junction}

\section{TO THE EDITORS:}

We appreciate the interest of Chalkiadakis and Ziogas in our article on the role of abdominal-transhiatal resection for adenocarcinoma of the esophagogastric junction and would like to underline the conclusions of our initial report. ${ }^{1,2}$ We completely agree that the role of high-volume surgeons and high-volume hospitals is critical for overall patient outcome, particularly in case of esophagogastric tumors. Main purpose of the study was to evaluate feasibility of the abdominal-transhiatal approach not only for type III cancer but for type II also, from a surgical perspective. However, even though at present no standard approach has been established, as we clearly stated in the
"Conclusion," attempts to further improve prognosis should include multimodal therapy. Moreover, we agree that in the near future it will be useful to provide genetic markers for a patient-tailored prevention and treatment strategy.

\section{Fabio Carboni, MD, PhD}

Department of Digestive Surgery, Regina Elena Cancer Institute, Rome, Italy

e-mail: fabiocarb@tiscali.it

Published Online: 25 April 2009

(C) Society of Surgical Oncology 2009

\section{REFERENCES}

1. Chalkiadakis GE, Ziogas D. Progress and limitations of surgery in improving outcomes of oesophagogastric junction cancer. Ann Surg Oncol. 2009.

2. Carboni F, Lorusso R, Santoro R, Lepiane P, Mancini P, Sperduti I, et al. Adenocarcinoma of the esophagogastric junction: the role of abdominal-transhiatal resection. Ann Surg Oncol. 2009;16:304312. 